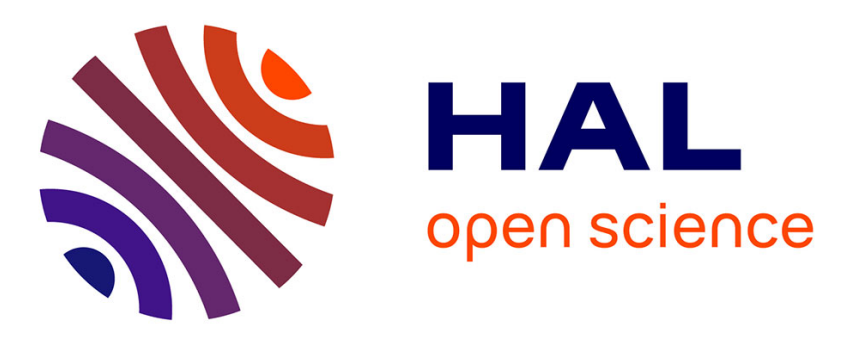

\title{
New trends in computational mechanics: model order reduction, manifold learning and data-driven
}

Jose Vicente Aguado, Domenico Borzacchiello, Elena Lopez, Emmanuelle Abisset-Chavanne, David González, Elías Cueto, Francisco Chinesta

\section{- To cite this version:}

Jose Vicente Aguado, Domenico Borzacchiello, Elena Lopez, Emmanuelle Abisset-Chavanne, David González, et al.. New trends in computational mechanics: model order reduction, manifold learning and data-driven. 9th Annual US-France symposium of the International Center for Applied Computational Mechanics, Jun 2016, Compiègne, France. hal-01878190

\author{
HAL Id: hal-01878190 \\ https://hal.science/hal-01878190
}

Submitted on 20 Sep 2018

HAL is a multi-disciplinary open access archive for the deposit and dissemination of scientific research documents, whether they are published or not. The documents may come from teaching and research institutions in France or abroad, or from public or private research centers.
L'archive ouverte pluridisciplinaire HAL, est destinée au dépôt et à la diffusion de documents scientifiques de niveau recherche, publiés ou non, émanant des établissements d'enseignement et de recherche français ou étrangers, des laboratoires publics ou privés. 


\section{New trends in computational mechanics : mo- del order reduction, manifold learning and data-driven}

\section{Jose Vicente Aguado ${ }^{1}$, Domenico Borzacchiello ${ }^{1}$, Elena Lopez $^{1}$, Em- manuelle Abisset-Chavanne ${ }^{1}$, David Gonzalez ${ }^{2}$, Elias Cueto ${ }^{2}$, Fran- cisco Chinesta ${ }^{1}$}

${ }^{1}$ High Performance Computing Institute \& ESI GROUP Chair

Ecole Centrale de Nantes, 1 rue de la Noe, 44300 Nantes, France

@ : Francisco.Chinesta@ec-nantes.fr

${ }^{2}$ I3A, Universidad de Zaragoza, Maria de Luna s/n, E-50018 Zaragoza, Spain

@:ecueto@unizar.es

ABSTRACT. Engineering sciences and technology is experiencing the data revolution. In the past models were more abundant than data, too expensive to be collected and analyzed at that time. However, nowadays, the situation is radically different, data is much more abundant (and accurate sometimes) than existing models, and a new paradigm is emerging in engineering sciences and technology. This paper retraces some incipient applications based on data within the framework of computational mechanics. Three main topics are addressed in the present work: (i) construction of solution manifolds and its use for interpolating new solutions on the manifold; (ii) constructing parametric solutions on the just extracted manifold; and (iii) defining behavior manifolds to perform data-driven simulation while avoiding the use of usual constitutive equations.

KEYWORDS: Model Order Reduction, Nonlinear Dimensionality Reduction, Vademecum, Machine Learning, Data-Driven 


\section{Introduction}

\subsection{The big picture}

Engineering sciences and technology, as any other branch of sciences and technology, is experiencing the data revolution. In the past models were more abundant than data, too expensive to be collected and analyzed at that time. However, nowadays, the situation is radically different, data is much more abundant (and accurate sometimes) than existing models, and a new paradigm is emerging in engineering sciences and technology.

Advanced model order reduction techniques allowed moving from data to information, because in many systems and despite the big amount of data the hidden information was quite reduced, and it was successfully extracted by applying many, nowadays state of the art model reduction techniques (POD, PGD or RB among many other variants).

Prior to solve a given problem the user must introduce the different involved parameters (e.g. material parameters and applied loads) as well as define the domain in which the problem is posed. However the just described procedure has a main handicap : it rarely allows proceeding in real-time. In those circumstances the real-time performance required in some applications are compromised. One could think that all these issues could be circumvented with the mere use of more powerful computers. Even if it could be a valuable route, it compromises the accessibility to the appropriate simulation resources of small and medium industries. In order to democratize simulation, new solutions are required. A possible alternative consists of calculating offline (using all the needed computational resources and computing time) a parametric solution containing the solution of all possible scenario, that is then particularized online using light computational facilities, as deployed devices, tablets or even smartphones, for performing efficient simulation, optimization, inverse analysis, uncertainty propagation and simulation-based control, all them under real-time constraints.

Even if someone could think for a while that for constructing the parametric solutions just announced it is enough to solve the model at hand for any possible choice of the parameters that it involves, it is clear that such a procedure rapidly fails because it involves a combinatorial explosion (e.g. ten parameters each one taking ten possible values will involve ten to the power of ten possibilities, and 10 parameters taking 10 possible values remains too simplistic in applications of practical interest).

Recently model order reduction opened new possibilities. First, Proper Orthogonal Decompositions - POD - allows extracting the most significant characteristics of the solution, that can be then applied for solving models slightly different to the ones that served to defined the reduced approximation basis, by simply projecting the searched solution onto the extracted reduced approximation basis [CHI16].

Another family of model reduction techniques lies in the used of Reduced Bases constructed by combining a greedy algorithm and "a priori" error indicators. It needs 
for some amount off-line work but then the reduced basis can be used on-line for solving different models with a perfect control of the solution accuracy because the availability of error bounds. When the error is inadmissible, the reduced basis can be enriched by invoking again the same greedy algorithm [CHI16].

Finally, Proper Generalized Decomposition methods are based on the use of separated representations [LAD85, LAD96, AMM06, AMM10]. Such separated representations are considered when solving at-hand partial differential equations by employing procedures based on the separation of variables, as described in the next section.

Advanced clustering techniques not only helps engineers and analysts, they become crucial in many areas where models, approximation bases, parameters, ... are adapted depending on the local (in space and time senses) state of the system. They makes possible define hierarchical and goal-oriented modeling.

Machine and manifold learning is also helping for extracting the manifold in which the solutions of complex and coupled engineering problems are living. Thus, uncorrelated parameters can be efficiently extracted from the collected data coming from numerical simulations, experiments or even from the data collected from adequate measurement devices. As soon as uncorrelated parameters are identified (constituting the information level), the solution of the problem can be predicted in new points of the parametric space, from adequate interpolations or even more, parametric solutions can be obtained within an adequate framework able to circumvent the curse of dimensionality (combinatorial explosion) for any value of the uncorrelated model parameters. Thus, the subtle circle is closed by linking data to information, information to knowledge and finally knowledge to real time decision-making, opening unimaginable possibilities within the so-called DDDAS (Dynamic Data Driven Application Systems) that allows even model-free simulations.

All the techniques just referred will be revisited in the present chapter.

\subsection{The PGD at glance}

Most of the existing model reduction techniques proceed by extracting a suitable reduced basis and then projecting on it the problem solution. Thus, the reduced basis construction precedes its use in the solution procedure, and one must be careful on the suitability of a particular reduced basis when employed for representing the solution of a particular problem. This issue disappears if the approximation basis is constructed at the same time that the problem is solved. Thus, each problem has its associated basis in which its solution is expressed. One could consider few terms in its approximation, leading to a reduced representation, or all the terms needed for approximating the solution up to a certain accuracy level. The Proper Generalized Decomposition (PGD) proceeds in this manner. 
When calculating the transient solution of a generic problem $u(x, t)$ we usually consider a given basis of space functions $N_{i}(x), i=1, \cdots, \mathcal{N}$, the so-called shape functions within the finite element framework, and approximate the problem solution as

$$
u(x, t) \approx \sum_{i=1}^{\mathcal{N}} a_{i}(t) N_{i}(x),
$$

that implies a space-time separated representation where the time-dependent coefficients $a_{i}(t)$ are unknown at each time (when proceeding incrementally) and the space functions $N_{i}(x)$ are given "a priori", e.g. polynomial basis. POD and Reduced Bases methodologies consider a reduced basis $\phi_{i}(x)$ for approximating the solution instead of using the generic functions $N_{i}(x)$. The former are expected to be more suitable for approximating the problem at-hand. Thus, it results

$$
u(x, t) \approx \sum_{i=1}^{R} b_{i}(t) \phi_{i}(x),
$$

where in general $R \ll \mathcal{N}$. Again (2) represents a space-time separated representation where the time-dependent coefficient must be calculated at each time during the incremental solution procedure.

Inspired from these results one could consider the general space-time separated representation

$$
u(x, t) \approx \sum_{i=1}^{N} X_{i}(x) T_{i}(t),
$$

where now neither the time-dependent functions $T_{i}(t)$ nor the space functions $X_{i}(x)$ are "a priori" known. Both will be computed on-the-flight when solving the problem.

As soon as one postulate that the solution of a transient problem can be expressed in the separated form (3) whose approximation functions $X_{i}(x)$ and $T_{i}(t)$ will be determined during the problem solution, one could make a step forward and assume that the solution of a multidimensional problem $u\left(x_{1}, \cdots, x_{d}\right)$ could be found in the separated form

$$
u\left(x_{1}, x_{2}, \cdots, x_{d}\right) \approx \sum_{i=1}^{N} X_{i}^{1}\left(x_{1}\right) X_{i}^{2}\left(x_{1}\right) \cdots X_{i}^{d}\left(x_{d}\right),
$$

and even more, expressing the 3D solution $u(x, y, z)$ as a finite sum decomposition involving lower dimensional functions

$$
u(x, y, z) \approx \sum_{i=1}^{N} X_{i}(x) Y_{i}(y) Z_{i}(z),
$$

or

$$
u(x, y, z) \approx \sum_{i=1}^{N} X_{i}(x, y) Z_{i}(z)
$$


and the solution of a parametric problem $u\left(\mathbf{x}, t, p_{1}, \cdots, p_{\wp}\right)$ as

$$
u\left(\mathbf{x}, t, p_{1}, \cdots, p_{\wp}\right) \approx \sum_{i=1}^{N} X_{i}(\mathbf{x}) T_{i}(t) \prod_{k=1}^{\wp} P_{i}^{k}\left(p_{k}\right) .
$$

The performances of all these separated representations are quite impressive in many cases.

For a review on such techniques and their applications in engineering sciences the interested reader can refer to [CHI10, CHI11, CHI13a, CHI13b] and the numerous references therein concerning the space-time / space-frequency decomposition [BOU97, LAD99, AMM07, AMM11, BOU13, RIO13, BAR14], space separation [BOG12, VID12, VID13, BOG14, BOR15] and parametric solutions [CHI13b, HEY13, AMM14, NER15], allowing real-time simulations [CHI13b, GON14, GON15], optimization [GHN11, CHI13b, CHI14, BOR16, AGU17], simulation-based control [GHN12, GON12, CHI13b, AGU15], uncertainty propagation [NOU08, NOU09a, NOU09b, NOU10] or multi-scale upscaling [LAM10, NER10, CRE13].

In all the applications referenced above the choice of parameters was a simple matter : material or process parameters, boundary conditions, etc ... However, in other applications the extraction of uncorrelated model parameters is not an easy task, as it is for example the case when addressing shape parametrization or the description of microstructures. These issues will be addressed in what follows.

\section{Constructing slow manifolds}

It is well known that microstructures or shapes do not allow simple reduced descriptions. The main question is not if microstructures or shapes define or not slow manifolds, the question is if they can or not be parametrized, i.e. represented, from slow manifolds. The same issue applies in visualization of high-dimensional data.

\subsection{From Principal Component Analysis - PCA - to Kernel Principal Component Analysis - kPCA}

Let us consider $D$ observed variables defining the vector $\mathbf{X} \in \mathbb{R}^{D}$. These are commonly referred to in the MOR literature as snapshots. We assume that these variables are therefore not uncorrelated and, notably, that there exists a linear transformation $\mathbf{W}$ defining the vector $\mathbf{Y} \in \mathbb{R}^{d}$, where $d<D$ represents the unknown so-called latent variables, according to

$$
\mathbf{X}=\mathbf{W Y}
$$

The transformation $\mathbf{W}, D \times d$, is assumed to verify the orthogonality condition $\mathbf{W}^{T} \mathbf{W}=\mathbf{I}_{d}$, where $\mathbf{I}_{d}$ represents the $d \times d$-identity matrix ( $\mathbf{W} \mathbf{W}^{T}$ is not necessarily $\mathbf{I}_{D}$ ). The existence of such a transformation is precisely at the origin of PCA methods. 
We assume the existence of $M$ different snapshots $\mathbf{X}_{1}, \ldots, \mathbf{X}_{M}$, that can be stored in the columns of the $D \times M$ matrix $\mathbb{X}$. The associated $d \times M$ reduced matrix $\mathbb{Y}$ contains the associated vectors $\mathbf{Y}_{i}, i=1, \ldots, M$.

PCA is able to calculate both $d$-the necessary number of members in the basis of the reduced-order subspace- and the transformation matrix $\mathbf{W}$. PCA proceeds by guaranteeing maximal preserved variance and decorrelation in the latent variable set. From a statistical point of view, therefore, it can be assumed that the latent variables are uncorrelated (no linear dependencies among them) or mutually orthogonal, thus constituting a basis. In practice, this means that the covariance matrix of $\mathbb{Y}$, defined as

$$
\mathbf{C}_{y y}=\mathrm{E}\left\{\mathbb{Y} \mathbb{Y}^{T}\right\}
$$

is diagonal. Thus, we consider

$$
\mathbf{C}_{x x}=\mathrm{E}\left\{\mathbb{X} \mathbb{X}^{T}\right\}=\mathrm{E}\left\{\mathbf{W} \mathbb{Y} \mathbb{Y}^{T} \mathbf{W}^{T}\right\}=\mathbf{W E}\left\{\mathbb{Y} \mathbb{Y}^{T}\right\} \mathbf{W}^{T}=\mathbf{W C}_{y y} \mathbf{W}^{T},
$$

that by pre-multiplying and post-multiplying by $\mathbf{W}^{T}$ and $\mathbf{W}$ respectively, and taking into account that $\mathbf{W}^{T} \mathbf{W}=\mathbf{I}$, leads to

$$
\mathbf{C}_{y y}=\mathbf{W}^{T} \mathbf{C}_{x x} \mathbf{W} .
$$

The covariance matrix $\mathbf{C}_{x x}$ can then be factorized by applying the singular value decomposition,

$$
\mathbf{C}_{x x}=\mathbf{V} \boldsymbol{\Lambda} \mathbf{V}^{T},
$$

with $\mathbf{V}$ containing the orthonormal eigenvectors and $\boldsymbol{\Lambda}$ the diagonal matrix containing the eigenvalues (non-negative real numbers), assumed in descending order.

Substituting the factorized expression of the covariance matrix (12) into Eq. (11) it results

$$
\mathbf{C}_{y y}=\mathbf{W}^{T} \mathbf{V} \Lambda \mathbf{V}^{T} \mathbf{W} .
$$

This equality holds only when the $d$ columns of $\mathbf{W}$ are taken collinear with $d$ columns of $\mathbf{V}$.

From a geometrical point of view, the columns of $\mathbf{V}$ indicate the directions in $\mathbb{R}^{D}$ that span the subspace of the latent variables. We illustrate this interpretation in Fig. 1 where at left we can appreciate points that apparently belongs to $\mathbb{R}^{2}$, however, it is easy to see that all this point belongs to a slow one-dimensional manifold. PCA find an alternative coordinate system given by $\mathbf{V}$ (axes in red) in which all these points are described from a single coordinate.

Nonlinear methods are often more powerful than linear ones, because the connection between the latent variables and the observed ones may be much richer than a simple matrix multiplication. This situation is sketched in Fig. 2 where it can be noticed that no-rotation allows to extract the one-dimensional slow manifold. Thus, PCA indicates that the different points belongs to a two-dimensional space, with the risk of 


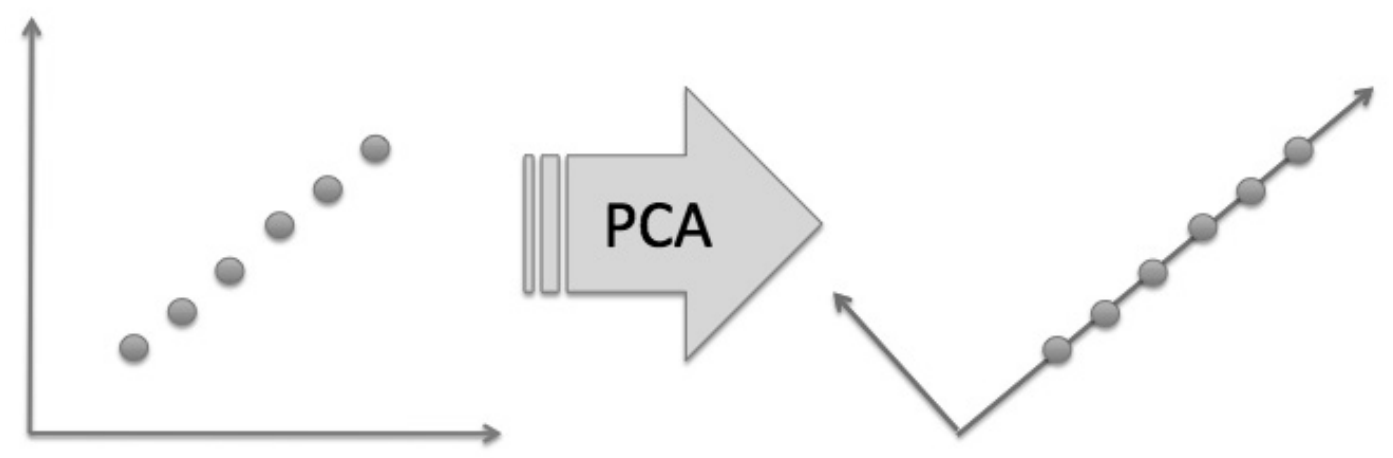

Figure 1. Geometrical interpretation of PCA
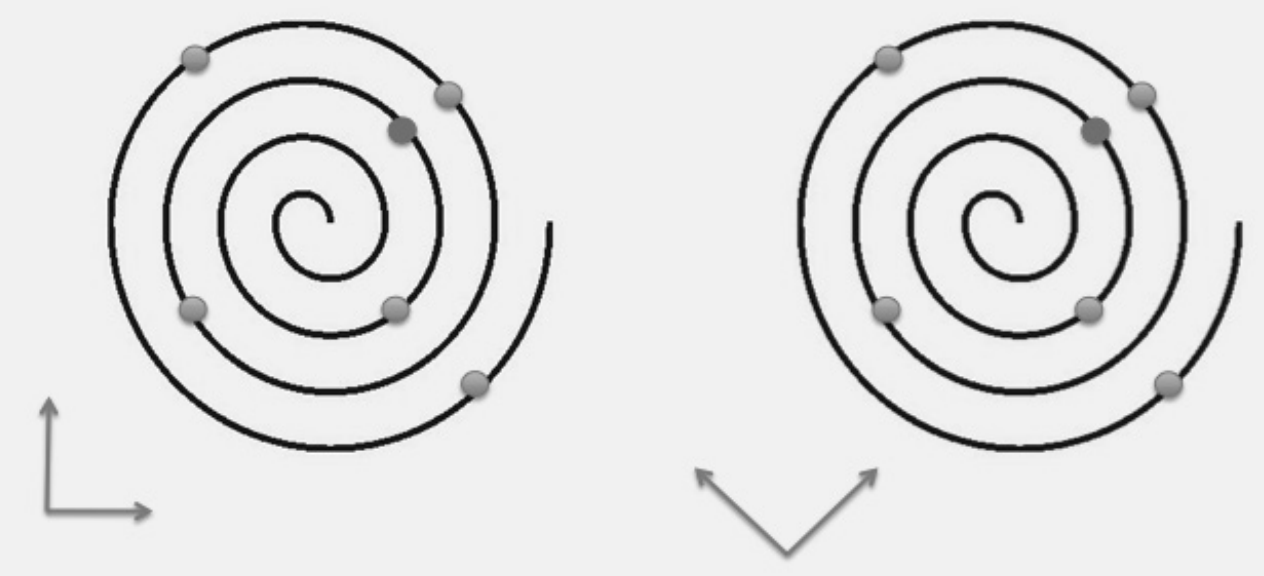

Figure 2. PCA limits in presence of strongly-nonlinear manifolds

concluding that the closest point (using the $2 \mathrm{D}$ euclidean distance) to the red point is in fact one that is very far from it when using the more appropriate geodesic distance on the one-dimensional slow manifold. Thus, the extraction of the slow manifold is compulsory and PCA is unable to accomplish the job.

These limitations justify the use of nonlinear dimensionality reduction techniques, as the local-PCA (IPCA), the kernel-PCA (kPCA) or the Locally Linear Embedding (LLE).

Local PCA applies standard PCA locally, that is, at each data-point and its closest neighbors. It is sketched in Fig. 3. The main issue related to its practical implementation is the alignment of the local bases unfolding the slow manifold, as discussed in many papers, e.g. [ZHA04]. 


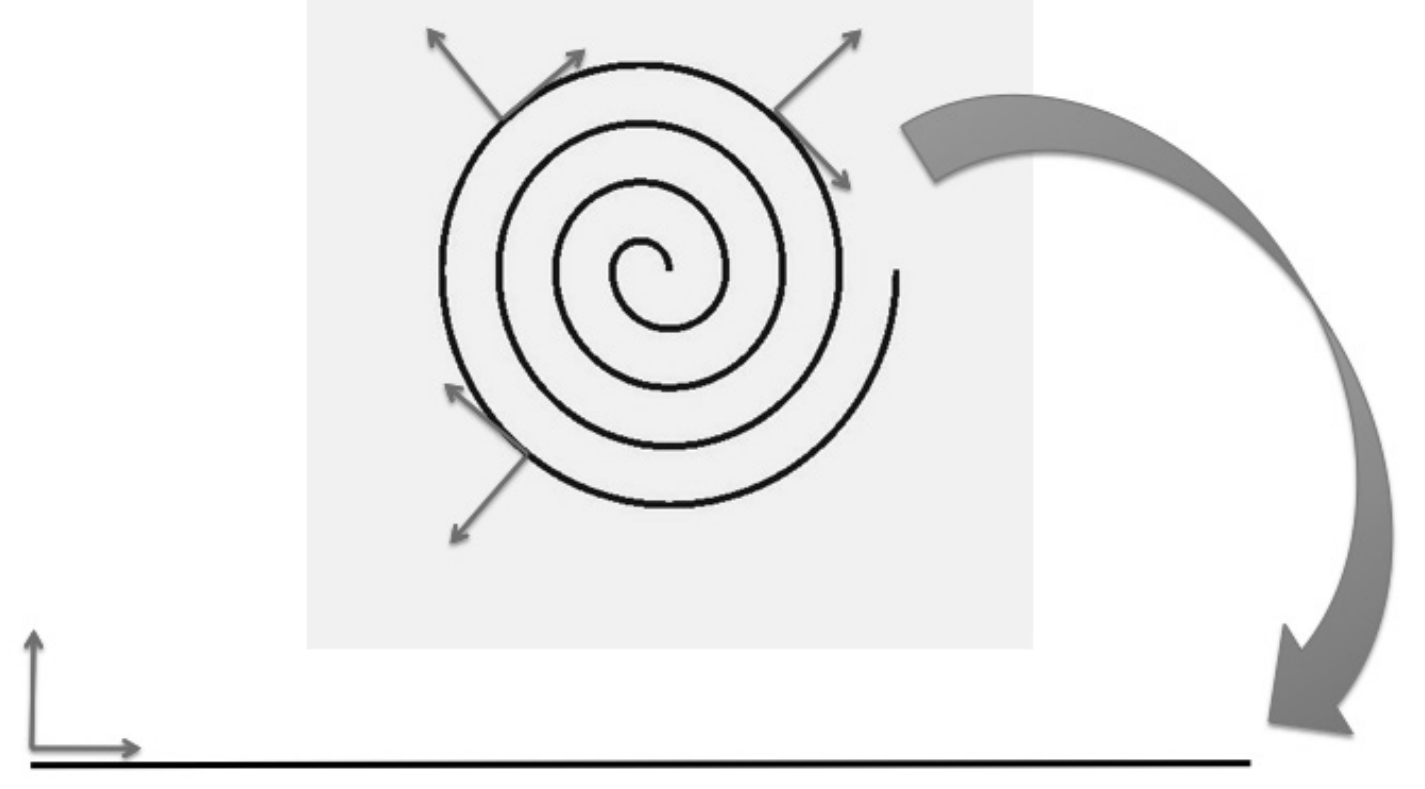

Figure 3. Scketch of local-PCA

\subsection{Kernel Principal Component Analysis (kPCA)}

PCA works with the sample covariance matrix, $\mathbb{X X}^{T}$. On the contrary, kPCA works with the matrix of pairwise scalar products that defines the Gram matrix $\mathbf{S}=$ $\mathbb{X}^{T} \mathbb{X}$ as it is also the case of Multidimensional Scaling (MDS) [LEE07]. In its classical version, MDS preserves pairwise scalar products instead of pairwise distances (both are closely related). It proceeds from

$$
\mathbf{S}=\mathbb{X}^{T} \mathbb{X}=\mathbb{Y}^{T} \mathbf{W}^{T} \mathbf{W} \mathbb{Y}=\mathbb{Y}^{T} \mathbb{Y}
$$

whose eigenvalue decomposition results

$$
\mathbf{S}=\mathbf{U} \boldsymbol{\Lambda} \mathbf{U}^{T}=\left(\mathbf{U} \boldsymbol{\Lambda}^{1 / 2}\right)\left(\boldsymbol{\Lambda}^{1 / 2} \mathbf{U}^{T}\right)=\left(\boldsymbol{\Lambda}^{1 / 2} \mathbf{U}^{T}\right)^{T}\left(\boldsymbol{\Lambda}^{1 / 2} \mathbf{U}^{T}\right)
$$

from which it results

$$
\mathbb{Y}=\mathbf{I}_{d \times M} \Lambda^{1 / 2} \mathbf{U}^{T}
$$

The idea behind kernel-PCA methods is simple : data not linearly separable in $D$ dimensions, could be linearly separated if previously projected to a space in $Q>D$ dimensions. Thus, surprisingly, kPCA begins by projecting the data to an even higher dimensional space. One the biggest advantages of this technique is that there is no need to explicitly determine the analytical expression of the mapping.

The symmetric matrix related to the mapped snapshots $\mathbb{Z}^{T} \mathbb{Z}$ has to be decomposed in eigenvalues and eigenvectors, after performing to the vectors involved in $\mathbb{Z}$ the double centering [LEE07]. 
Now, the eigenvalue-eigenvector decomposition can be performed according to

$$
\mathbb{Z}^{T} \mathbb{Z}=\mathbf{U} \boldsymbol{\Lambda} \mathbf{U}^{T},
$$

from which it results

$$
\mathbb{Y}=\mathbf{I}_{d \times M} \boldsymbol{\Lambda}^{1 / 2} \mathbf{U}^{T} .
$$

It is worth noting that the previous procedure only needs scalar products in the intermediate space $\mathbb{R}^{Q}$. The Mercer's theorem allows computing such scalar products in the original space $\mathbb{R}^{D}$, by using the so-called kernel-trick. There are many possible choices [LEE07] and then the model calibration becomes its principal advantage and at the same time its main drawback.

\subsection{Locally Linear Embedding - LLE}

First we assume the existence of $M$ multi-dimensional data $\mathbf{X}_{m}, m=1, \ldots, M$, defined in a space of dimension $D$, i.e. $\mathbf{X}_{m} \in \mathbb{R}^{D}$. LLE proceeds as follows [ROW00] :

- Each point $\mathbf{X}_{m}, m=1, \ldots, M$ is linearly reconstructed from its $K$-nearest neighbors. In principle $K$ should be greater that the expected dimension $d$ of the underlying embedded slow manifold and the points should be close enough to ensure the validity of the linear approximation. In general, a large-enough number of neighbors $K$ and a large-enough sampling $M$ ensures a satisfactory reconstruction. For each point $\mathbf{X}_{m}$ we can write the locally linear data reconstruction as :

$$
\mathbf{X}_{m}=\sum_{i \in \mathcal{S}_{m}} W_{m i} \mathbf{X}_{i},
$$

where $W_{m i}$ are the unknown weights and $\mathcal{S}_{m}$ the set of the $K$-nearest neighbors of $\mathbf{X}_{m}$. As the same weights appears in different locally linear reconstructions, the best compromise is searched by looking for the weights, all them grouped in vector $\mathbf{W}$, that minimize the functional

$$
\mathcal{F}(\mathbf{W})=\sum_{m=1}^{M}\left\|\mathbf{X}_{m}-\sum_{i=1}^{M} W_{m i} \mathbf{X}_{i}\right\|^{2}
$$

where here $W_{m i}$ is zero if $\mathbf{X}_{i}$ does not belong to the set of $K$-nearest neighbors of $\mathbf{X}_{m}$. The minimization of $\mathcal{F}(\mathbf{W})$ allows to determine all the weights involved in all the locally linear data reconstruction.

- We suppose now that each linear patch around $\mathbf{X}_{m}, \forall m$, is mapped into a lower dimensional embedding space of dimension $d, d \ll D$. Because of the linear mapping of each patch, weights remain unchanged. The problem becomes the determination of the coordinates of each point $\mathbf{X}_{m}$ when it is mapped into the low dimensional space, $\mathbf{Y}_{m} \in \mathbb{R}^{d}$. 
For this purpose a new functional $\mathcal{G}$ is introduced, that depends on the searched coordinates $\mathbf{Y}_{1}, \ldots, \mathbf{Y}_{M}$ :

$$
\mathcal{G}\left(\mathbf{Y}_{1}, \ldots, \mathbf{Y}_{M}\right)=\sum_{m=1}^{M}\left\|\mathbf{Y}_{m}-\sum_{i=1}^{M} W_{m i} \mathbf{Y}_{i}\right\|^{2}
$$

where now the weights are known and the reduced coordinates $\mathbf{Y}_{m}$ are unknown. The minimization of functional $\mathcal{G}$ results in a $M \times M$ eigenvalue problem whose $d$ bottom non-zero lowest eigenvalues define the set of orthogonal coordinates in which the manifold is mapped.

The use of LLE exhibits some weakness, the first related to the use of euclidian distances, even if other choices could be considered. The second is related to a covariance normalization considered when solving the second eigenproblem above. Alternatives, like the t-SNE [MAA08] allows circumventing the last difficulty.

\subsection{Discussion}

The main advantage of local-PCA is that it allows extracting the real local reduced dimensionality and the fact of having a real geometrical transformation allowing not only extracting the embedded manifold but also to map points outside the slow manifold. Moreover, PCA-based transformations preserve distances, where other nonlinear dimensionality reduction strategies fail to accomplish it.

\section{Manifold-learning-based computational mechanics}

Imagine different physical systems characterized by vectors $\mathbf{X}_{m} \in \mathbb{R}^{D}$, whose associated solutions (of the physical problem at hand) are dented by $\mathbf{T}_{m}$. By applying the LLE (among other possible choices) the slow manifold is extracted. Now, when considering a new physical system, characterized by $\mathbf{X}$, by applying the LLE it results its image $\mathbf{Y}$ on the manifold, with the weights associated to its reconstruction, i.e.

$$
\mathbf{Y}=\sum_{i \in \mathcal{S}(\mathbf{X})} W_{i} \mathbf{Y}_{i},
$$

where $\mathcal{S}(\mathbf{X})$ represents the set of the K-nearest neighbors of $\mathbf{X}$. Now, a prediction of the problem solution $\mathbf{T}$ writes

$$
\mathbf{T}=\sum_{i \in \mathcal{S}(\mathbf{X})} W_{i} \mathbf{T}_{i}
$$

This strategy was successfully considered in [LOP16] for addressing models involving parametrized microstructures and shapes. However, there is a strong assumption 
in the rationale just described. The neighbors and their associated weights in the parametric space are consider to interpolate the solution.

A more accurate approach consists of calculating the parametric solution within for example the PGD framework,

$$
T\left(\mathbf{x}, t, y_{1}, \cdots, y_{d}\right) \approx \sum_{i=1}^{N} \mathcal{X}_{i}(\mathbf{x}) \mathcal{T}_{i}(t) \mathcal{Y}_{i}^{1}\left(y_{1}\right) \cdots \mathcal{Y}_{i}^{d}\left(y_{d}\right),
$$

where here $\mathbf{x}$ denotes the space coordinates involved in usual models and their associated partial differential equations, $t$ the time involved in transient models and $y_{j}$ are the latent variables grouped in vector $\mathbf{Y}$ (defining the slow manifold). This procedure was successfully applied in [GON16] for addressing the same problems that were addressed in [LOP16].

\section{Data-Driven simulations}

We consider mechanical tests conducted on a perfectly elastic material, in a specimen exhibiting uniform stresses and strains. For a while, we do not consider issues related to data generation and collection, they will addressed later. More complex behaviors were addressed in [IBA16]. Thus, for $M$ randomly applied external loads, we assume ourselves able to collect $M$ couples $\left(\boldsymbol{\sigma}_{m}, \boldsymbol{\epsilon}_{m}\right), m=1, \ldots, M$. Each stressstrain couple could be represented as a single point $\mathbf{P}_{m}$ in a phase space of dimension $D=12$ (the six distinct components of the stress and strain tensors, respectively). In the sequel Voigt notion will be considered, i.e. stress and strain tensors will be represented as vectors and consequently the fourth-order elastic tensor reduces to a $6 \times 6$ square matrix.

Each vector $\mathbf{P}_{m}$ thus defines a point in a space of dimension $D$ and, therefore, the whole set of samples represents a set of $M$ points in $\mathbb{R}^{D}$. We conjecture that all these points belong to (or can be embedded into) a certain low-dimensional manifold embedded into the high-dimensional space $\mathbb{R}^{D}$ allowing for a nonlinear dimensionality reduction as discussed in [IBA16]. In what follows we proceeds without such a dimensionality reduction and consider the simplest strategy proposed and discussed in [IBA16]. We consider locally linear approximations, that allow writing

$$
\mathbf{P}_{m}=\sum_{i=1}^{M} W_{m i} \mathbf{P}_{i}
$$

with $W_{m i}=0$ if $i \notin \mathcal{S}_{m}$ (set containing the $K$-nearest neighbors of $\mathbf{P}_{m}$ ). By minimizing the functional

$$
\mathcal{H}(\mathbf{C})=\sum_{i \in \mathcal{S}_{m}}\left(\boldsymbol{\sigma}_{i}-\mathbf{C} \boldsymbol{\epsilon}_{i}\right)^{2} .
$$

we obtain the secant elastic behavior $\mathbf{C}\left(\mathbf{P}_{m}\right) \equiv \mathbf{C}_{m}$. 


\subsection{Data-based weak form}

From the just identified locally linear behavior $\mathbf{C}(\mathbf{P})$ one could apply the simplest linearization technique operating on the standard weak form

$$
\int_{\Omega} \boldsymbol{\epsilon}^{*}(\mathbf{x}): \boldsymbol{\sigma}(\mathbf{x}) d \mathbf{x}=\int_{\Gamma_{N}} \mathbf{u}^{*}(\mathbf{x}) \cdot \mathbf{t}(\mathbf{x}) d \mathbf{x},
$$

where at each point, from the stress-strain couple at position $\mathbf{x}, \mathbf{P}(\mathbf{x})$, the locally linear behavior $\mathbf{C}(\mathbf{P}(\mathbf{x}))$ can be obtained (in practice at the Gauss points used for the integration of the weak form) that allows us to write, using Voigt notation

$$
\int_{\Omega} \boldsymbol{\epsilon}^{*}(\mathbf{x}) \cdot(\mathbf{C}(\mathbf{x}) \boldsymbol{\epsilon}(\mathbf{x})) d \mathbf{x}=\int_{\Gamma_{N}} \mathbf{u}^{*}(\mathbf{x}) \cdot \mathbf{t}(\mathbf{x}) d \mathbf{x} .
$$

This allows, in turn, to compute the displacement field and from it, to update the strain and stress fields, and compute again the locally linear behavior. The process continues until convergence. The discretization related to other two alternative descriptions was deeply considered in [IBA16].

\subsection{Constructing the constitutive manifold}

In the sequel we consider perfectly elastic isotropic behavior, i.e. inelastic (irreversible) deformations are neglected, and also assume small displacements and deformations. We consider the mechanical specimen occupying the domain $\Omega \in \mathbb{R}^{3}$, of boundary $\Gamma \equiv \partial \Omega$ with prescribed displacements on $\Gamma_{D}, \mathbf{u}\left(\mathbf{x} \in \Gamma_{D}\right)=\mathbf{u}_{g}$, without loss of generality assumed vanishing, i.e. $\mathbf{u}_{g}=\mathbf{0}$, and prescribed tractions in the complementary boundary $\Gamma_{N}\left(\Gamma=\Gamma_{D} \cup \Gamma_{N}\right),\left.\boldsymbol{\sigma} \cdot \mathbf{n}\right|_{\mathbf{x} \in \Gamma_{N}}=\mathbf{t}$. Boundary $\Gamma_{N}$ is at its

turn decomposed in the free-traction region $\Gamma_{N}^{f}$ where $\mathbf{t}=\mathbf{0}$ and the remaining part $\Gamma_{N}^{t}$ where non null external tractions, $\mathbf{t} \neq \mathbf{0}$, apply.

We also ignore mass and inertia contributions to the mechanical state and assume that in absence of external tractions, i.e. when $\mathbf{t}=\mathbf{0}$, the mechanical part remains free of strains and stresses, i.e. $\boldsymbol{\epsilon}=\mathbf{0}$ and $\boldsymbol{\sigma}=\mathbf{0}$ respectively.

\subsubsection{Linear elastic behavior}

The external traction $\mathbf{t}$ is applied, from which the equilibrium reads

$$
\int_{\Omega} \boldsymbol{\epsilon}^{*}(\mathbf{x}) \cdot \boldsymbol{\sigma}(\mathbf{x}) d \mathbf{x}=\int_{\Gamma_{N}} \mathbf{u}^{*}(\mathbf{x}) \cdot \mathbf{t} d \mathbf{x} .
$$

Taking into account the strain- and stress-free reference configuration previously discussed, the problem can be expressed in the incremental form

$$
\int_{\Omega} \Delta \boldsymbol{\epsilon}^{*}(\mathbf{x}) \cdot \Delta \boldsymbol{\sigma}(\mathbf{x}) d \mathbf{x}=\int_{\Gamma_{N}} \Delta \mathbf{u}^{*}(\mathbf{x}) \cdot \mathbf{t} d \mathbf{x},
$$


or introducing the linear behavior

$$
\int_{\Omega} \Delta \boldsymbol{\epsilon}^{*}(\mathbf{x}) \cdot(\mathbf{C} \Delta \boldsymbol{\epsilon}(\mathbf{x})) d \mathbf{x}=\int_{\Gamma_{N}} \Delta \mathbf{u}^{*}(\mathbf{x}) \cdot \mathbf{t} d \mathbf{x}
$$

where the tangent matrix $\mathbf{C}$ is unknown, but because the linear elastic behavior remains constant everywhere in the domain. Using a parametrization of symmetric $6 \times 6$ matrices (the more general one making use of canonical matrices with a single nonzero entry, taking a unit value) we can write

$$
\mathbf{C}=\sum_{i=1}^{\mathcal{K}} \alpha_{i} \mathbf{K}_{i}
$$

with coefficients $\alpha_{i}$ unknown. When using canonical matrices $\mathcal{K}=21$, and any symmetric matrix can be written from a linear combination of those 21 canonical matrices by considering adequate coefficients $\alpha_{i}, i=1, \cdots, 21$.

By introducing this tangent matrix representation into the equilibrium weak form it results

$$
\int_{\Omega} \Delta \boldsymbol{\epsilon}^{*}(\mathbf{x}) \cdot\left(\left(\sum_{i=1}^{\mathcal{K}} \alpha_{i} \mathbf{M}_{i}\right) \Delta \boldsymbol{\epsilon}(\mathbf{x})\right) d \mathbf{x}=\int_{\Gamma_{N}} \boldsymbol{\Delta} u^{*}(\mathbf{x}) \cdot \mathbf{t} d \mathbf{x}
$$

whose discrete form reads

$$
\Delta \mathbf{U}^{*} \cdot\left(\sum_{i=1}^{\mathcal{K}} \alpha_{i} \mathbf{K}_{i}\right) \Delta \mathbf{U}=\Delta \mathbf{U}^{*} \cdot \mathbf{T}
$$

with $\mathbf{K}_{i}$ the rigidity matrices corresponding to the canonical behaviors.

We assume that local displacement and their associated strains are accessible on a certain region of the domain whose associated degrees of freedom are indicated with the superscript $\bullet \mathcal{O}$. Thus, making use of a partition of the displacements $\Delta \mathbf{U}^{\mathcal{O}}$ and $\Delta \mathbf{U}^{\mathcal{H}}$ referring to the observable and hidden displacements respectively, the previous discrete nonlinear system can be solved to compute the unknown displacements $\Delta \mathbf{U}^{\mathcal{H}}$ and material coefficients $\alpha_{i}$.

When considering a linear behavior the resulting displacements, strains and stresses can be easily derived from $\mathbf{U} \equiv \Delta \mathbf{U}=\left(\Delta \mathbf{U}^{\mathcal{H}}, \Delta \mathbf{U}^{\mathcal{O}}\right)^{T}$ by considering

$$
\left\{\begin{array}{l}
\mathbf{u}(\mathbf{x})=\sum_{i=1}^{\mathcal{N}} \mathbf{U}_{i} N_{i}(\mathbf{x}) \\
\boldsymbol{\epsilon}=\nabla_{S} \mathbf{u} \\
\boldsymbol{\sigma}=\mathbf{C} \boldsymbol{\epsilon}
\end{array}\right.
$$


where $\mathcal{N}$ is the number of nodes considered for approximating the displacement field $\mathbf{u}(\mathbf{x})$ and $N_{i}(\mathbf{x})$ the associated shape functions. $\nabla_{S} \bullet$ denotes the symmetric component of the gradient operator and $\mathbf{C}$ results from $\alpha_{i}$

$$
\mathbf{C}=\sum_{i=1}^{\mathcal{K}} \alpha_{i} \mathbf{K}_{i} .
$$

\subsubsection{Nonlinear elastic behavior}

In the nonlinear case, when performing homogeneous tests, it suffices applying the external load incrementally and at each step, being the behavior the same everywhere in the tested coupon, the behavior will be identified incrementally. However, such a procedure do not allow exploring the whole strain-stress space, there are not testing facilities able to prescribe any complex multi-axial strain. In our recent works we propose an alternative data-driven inverse procedure that more than using homogeneous tests, exploits complex tests to cover as much as possible the behavioral manifold. This approach combines an incremental loading and a clustering to identify the candidate points where the behavior should be updated from the inverse procedure at each loading step.

\section{Data-driven upscaling of viscous flows in porous media}

Isothermal flows of complex fluids in complex microstructures can be simulated by solving the momentum and mass balance equations and a suitable rheological constitutive model. For inertialess incompressible flows, these balance equations read,

$$
\nabla \cdot \boldsymbol{\sigma}=\mathbf{0},
$$

and

$$
\nabla \cdot \mathbf{v}=0,
$$

respectively. Here, $\sigma$ is the Cauchy stress tensor and $\mathbf{v}$ the velocity field, both defined at time $t$ at each point within the fluid domain $\Omega_{f}$. When considering porous media, the domain $\Omega$ is assumed fully saturated, with the fluid phase occupying the region $\Omega_{f}$ whereas the remaining part $\Omega_{s}=\Omega-\Omega_{f}$ is occupied by a solid phase assumed at rest.

An appropriate constitutive equation must be postulated to describe the fluid's rheology. There are many possible choices, the most usual ones being related to Newtonian and the generalized-Newtonian summarized below.

For a Newtonian fluid, the constitutive equation reads

$$
\boldsymbol{\sigma}=-p \mathbf{I}+\boldsymbol{\tau}=-p \mathbf{I}+2 \eta \mathbf{D},
$$

where $p$ is the pressure field that can be interpreted as the Lagrange multiplier associated with the incompressibility constraint, $\mathbf{I}$ is the identity tensor, $\boldsymbol{\tau}$ the extra-stress 
tensor, $\eta$ the constant fluid viscosity and $\mathbf{D}$ the rate of strain tensor, i.e. the symmetric part of the velocity gradient, $2 \mathbf{D}=\nabla \mathbf{v}+(\nabla \mathbf{v})^{T}$.

For a generalized-Newtonian fluid, the constitutive equation (39) remains formally unchanged but now the viscosity $\eta$ depends on the effective strain rate $\dot{\gamma}$ usually expressed from the second invariant of the rate of strain tensor, i.e. $\dot{\gamma}=\sqrt{2 \mathbf{D}: \mathbf{D}}$. The simplest of such models is the power-law (shear-thinning) viscosity given by

$$
\eta=\kappa \dot{\gamma}^{n-1},
$$

where $\kappa$ and $n$ are the consistency and power-law index, respectively. The value $n=1$ corresponds to a Newtonian fluid.

\subsection{Upscaling Newtonian and generalized-Newtonian fluids flowing in porous media}

The upscaling procedure was deeply addressed in our former works [LOP15, LOP16b, AMM16] to handle Newtonian, generalized-Newtonian fluid and quasi-Newtonian fluids.

For all these fluids, the flow model is solved in the representative volume $\omega(\mathbf{X})$ located at potion $\mathbf{X} \in \Omega$, where two phases coexist, i.e. the fluid phase occupying the domain $\omega_{f}(\mathbf{X})$ and the solid phase, assumed rigid and at rest, occupying the region $\omega_{s}(\mathbf{X})$, with $\omega_{f}(\mathbf{X}) \cup \omega_{s}(\mathbf{X})=\omega(\mathbf{X})$ and $\omega_{f}(\mathbf{X}) \cap \omega_{s}(\mathbf{X})=\emptyset$. The flow model consists of the mass and momentum balances complemented by the constitutive equation discussed in the previous section,

$$
\left\{\begin{array}{l}
\nabla \cdot \boldsymbol{\sigma}=\mathbf{0} \\
\nabla \cdot \mathbf{v}=0 \\
\boldsymbol{\sigma}=-p \mathbf{I}+2 \eta \mathbf{D}=-p \mathbf{I}+\boldsymbol{\tau}
\end{array},\right.
$$

with the viscosity $\eta$ constant in the case of Newtonian fluids or depending on the effective strain rate in the case of generalized-Newtonian fluids. The flow model above is complemented with the boundary condition $\mathbf{v}(\mathbf{x} \in \partial \omega(\mathbf{X}))=\mathbf{V}$, where $\mathbf{V}$ comes from the macroscopic flow problem.

The solution of the flow problem (41) allows calculating the velocity field $\mathbf{v}(\mathbf{x} \in$ $\left.\omega_{f}(\mathbf{X})\right)$, from it the strain rate $\mathbf{D}\left(\mathbf{x} \in \omega_{f}(\mathbf{X})\right)$, the local viscosity $\eta\left(\mathbf{x} \in \omega_{f}(\mathbf{X})\right)$ and finally the extra-stress tensor $\boldsymbol{\tau}\left(\mathbf{x} \in \omega_{f}(\mathbf{X})\right)$, fields that allow calculating the dissipated power in the RVE, $\mathcal{D} \mathcal{P}(\mathbf{V} ; \mathbf{X})$, associated with the prescribed macroscopic velocity $\mathbf{V}$ on its boundary $\partial \omega$

$$
\mathcal{D P}(\mathbf{V} ; \mathbf{X})=\int_{\omega_{f}(\mathbf{X})} \boldsymbol{\sigma}(\mathbf{x}): \mathbf{D}(\mathbf{x}) d \mathbf{x},
$$

with the specific microscopic dissipation $\mathcal{D} \mathcal{P}^{m}$ obtained by dividing $\mathcal{D P}$ given by (42) by the RVE volume $|\omega(\mathbf{X})|$. 
Obviously, being the model purely viscous it only involves dissipated power, and consequently the effective macroscopic model should account for that dissipated power. When considering the Darcy's model, the specific macroscopic dissipated power $\mathcal{D} \mathcal{P}^{M}$ reads

$$
\mathcal{D} \mathcal{P}^{M}(\nabla P, \mathbf{V})=\nabla P \cdot \mathbf{V},
$$

where by equating the micro and macro dissipations, it results

$$
\mathcal{D P}^{m}=\left.\nabla P\right|_{\mathbf{X}} \cdot \mathbf{V}(\mathbf{X}),
$$

or by assuming an effective permeability $\mathbf{K}_{e f f}(\mathbf{X})$

$$
\left.\nabla P\right|_{\mathbf{X}}=\mathbf{K}_{e f f}^{-1}(\mathbf{X}) \mathbf{V}(\mathbf{X}),
$$

from which it finally results

$$
\mathcal{D} \mathcal{P}^{m}(\mathbf{V} ; \mathbf{X})=\mathbf{K}_{e f f}^{-1}(\mathbf{X}):(\mathbf{V}(\mathbf{X}) \otimes \mathbf{V}(\mathbf{X}))
$$

The previous expression constitutes a constructive definition of the effective permeability. For calculating the last it suffices taking the second derivatives of $\mathcal{D} \mathcal{P}^{m}(\mathbf{V})$ related to the microstructure existing at location $\mathbf{X}$,

$$
\mathbf{K}_{e f f}^{-1}(\mathbf{X})=\frac{d^{2} \mathcal{D} \mathcal{P}^{m}(\mathbf{V} ; \mathbf{X})}{d \mathbf{V}^{2}}
$$

In the case of a Newtonian fluid, the velocity, strain-rate and stress fields scale linearly with the prescribed velocity on the RVE boundary and consequently the dissipated power scales with the square of the velocity, leading to a constant permeability, as deeply discussed in [LOP15].

\section{Conclusions}

We are not at the beginning of the end, but at the end of the beginning ! Data is expected enriching modeling approaches and even replacing too poor models in order to improve predictions accuracy. The big amount of collected data, including synthetic data generated from simulations, should perform bringing data, information, knowledge and decision making, operating under real-time constraints.

\section{Bibliographie}

[AGU15] J.V. Aguado, A. Huerta, F. Chinesta, E. Cueto. Real-time monitoring of thermal processes by reduced order modelling. International Journal for Numerical Methods in Engineering, 102/5, 991-1017, 2015.

[AGU17] J.V. Aguado, D. Borzacchiello, C. Ghnatios, F. Lebel, R. Upadhyay, C. Binetruy, F. Chinesta. A Simulation App based on reduced order modeling for manufacturing optimization of composite outlet guide vanes. Model. and Simul. in Eng. Sci., 4 :1, 2017, DOI 10.1186/s40323-017-0087-y 
[AMM06] A. Ammar, B. Mokdad, F. Chinesta, R. Keunings. A new family of solvers for some classes of multidimensional partial differential equations encountered in kinetic theory modeling of complex fluids. Journal of Non-Newtonian Fluid Mechanics, 139, 153-176, 2006.

[AMM07] A. Ammar, B. Mokdad, F. Chinesta, R. Keunings. A new family of solvers for some classes of multidimensional partial differential equations encountered in kinetic theory modeling of complex fluids. Part II : Transient simulation using space-time separated representation. Journal of Non-Newtonian Fluid Mechanics, 144, 98-121, 2007.

[AMM10] A. Ammar, F. Chinesta, A. Falco. On the convergence of a greedy rank-one update algorithm for a class of linear systems. Archives of Computational Methods in Engineering, 17/4, 473-486, 2010.

[AMM11] A. Ammar, F. Chinesta, E. Cueto, M. Doblare. Proper Generalized Decomposition of time-multiscale models. International Journal for Numerical Methods in Engineering, 90/5, 569-596, 2011.

[AMM14] A. Ammar, A. Huerta, F. Chinesta, E. Cueto, A. Leygue. Parametric solutions involving geometry : A step towards efficient shape optimization. Computer Methods in Applied Mechanics and Engineering, 268C, 178-193, 2014.

[AMM16] A. Ammar, E. Abisset-Chavanne, F. Chinesta, R. Keunings. Flow modelling of quasi-Newtonian fluids in two-scale fibrous fabrics. Advanced simulations. International Journal of Material Forming, In press, DOI 10.1007/s12289-016-1300-0.

[BAR14] A. Barbarulo, H. Riou, L. Kovalevsky, P. Ladeveze. PGD-VTCR : a reduced order model technique to solve medium frequency broad band problems on complex acoustical systems. Journal of Mechanical Engineering, 60/5, 307-314, 2014.

[BOG12] B. Bognet, A. Leygue, F. Chinesta, A. Poitou, F. Bordeu. Advanced simulation of models defined in plate geometries : 3D solutions with 2D computational complexity. Computer Methods in Applied Mechanics and Engineering, 201, 1-12, 2012.

[BOG14] B. Bognet, A. Leygue, F. Chinesta. Separated representations of 3D elastic solutions in shell geometries. Advanced Modelling and Simulation in Engineering Sciences, 2014, $1: 4$, http ://www.amses-journal.com/content/1/1/4

[BOR15] F. Bordeu, Ch. Ghnatios, D. Boulze, B. Carles, D. Sireude, A. Leygue, F. Chinesta. Parametric 3D elastic solutions of beams involved in frame structures. Advances in Aircraft and Spacecraft Science, 2/3, 233-248, 2015.

[BOR16] D. Borzacchiello, J.V. Aguado, F. Chinesta. Reduced Order Modelling for efficient numerical optimisation of a hot-wall Chemical Vapour Deposition reactor. International Journal of Numerical Methods for Heat and Fluid Flow, 27/4, DOI 10.1108/HFF-04-20160153, 2016.

[BOU97] P.A. Boucard, P. Ladeveze, M. Poss, P. Rougée. A non-incremental approach for large displacement problems. Computers \& Structures, 64, 499-508, 1997.

[BOU13] L. Boucinha, A. Gravouil, A. Ammar. Space-time proper generalized decompositions for the resolution of transient elastodynamic models. Computer Methods in Applied Mechanics and Engineering, 255, 67-88, 2013.

[CHI10] F. Chinesta, A. Ammar, E. Cueto. Recent advances and new challenges in the use of the Proper Generalized Decomposition for solving multidimensional models. Archives of Computational Methods in Engineering, 17/4, 327-350, 2010 b.

[CHI11] F. Chinesta, P. Ladeveze, E. Cueto. A short review in model order reduction based on Proper Generalized Decomposition. Archives of Computational Methods in Engineering, 
18, 395-404, 2011.

[CHI13a] F. Chinesta, R. Keunings, A. Leygue. The Proper Generalized Decomposition for advanced numerical simulations. A primer. Springerbriefs, Springer, 2013.

[CHI13b] F. Chinesta, A. Leygue, F. Bordeu, J.V. Aguado, E. Cueto, D. Gonzalez, I. Alfaro, A. Ammar, A. Huerta. Parametric PGD based computational vademecum for efficient design, optimization and control. Archives of Computational Methods in Engineering, 20/1, 31-59, 2013.

[CHI14] F. Chinesta, A. Leygue, B. Bognet, Ch. Ghnatios, F. Poulhaon, F. Bordeu, A. Barasinski, A. Poitou, S. Chatel, S. Maison-Le-Poec. First steps towards an advanced simulation of composites manufacturing by automated tape placement. International Journal of Material Forming, 7/1, 81-92, 2014.

[CHI16] F. Chinesta, A. Huerta, G. Rozza, K. Willcox K. Model Order Reduction, In the Encyclopedia of Computational Mechanics, 2nd Edition, Wiley, 2016.

[CRE13] M. Cremonesi, D. Neron, P.A. Guidault. P. Ladeveze. A PGD-based homogenization technique for the resolution of nonlinear multiscale problems. Computer Methods in Applied Mechanics and Engineering, 267, 275-292, 2013.

[GHN11] Ch. Ghnatios, F. Chinesta, E. Cueto, A. Leygue, P. Breitkopf, P. Villon. Methodological approach to efficient modeling and optimization of thermal processes taking place in a die : Application to pultrusion. Composites Part A, 42, 1169-1178, 2011.

[GHN12] Ch. Ghnatios, F. Masson, A. Huerta, E. Cueto, A. Leygue, F. Chinesta. Proper Generalized Decomposition based dynamic data-driven control of thermal processes. Computer Methods in Applied Mechanics and Engineering, 213, 29-41, 2012.

[GON12] D. Gonzalez, F. Masson, F. Poulhaon, A. Leygue, E. Cueto, F. Chinesta. Proper Generalized Decomposition based dynamic data-driven inverse identification. Mathematics and Computers in Simulation, 82/9, 1677-1695, 2012.

[GON14] D. Gonzalez, E. Cueto, F. Chinesta. Real-time direct integration of reduced solid dynamics equations. International Journal for Numerical Methods in Engineering, 99/9, 633-653, 2014.

[GON15] D. Gonzalez, I. Alfaro, C. Quesada, E. Cueto, F. Chinesta. Computational vademecums for the real-time simulation of haptic collision between nonlinear solids. Computer Methods in Applied Mechanics and Engineering, 283, 210-223, 2015.

[GON16] D. Gonzalez, J.V. Aguado, E. Abisset-Chavanne, E. Cueto, F. Chinesta. kPCA-based parametric solutions within the PGD framework, Archives of Computational Methods in Engineering, DOI 10.1007/s11831-016-9173-4, 2016.

[HEY13] Ch. Heyberger, P.A. Boucard, D. Neron. A rational strategy for the resolution of parametrized problems in the PGD framework. Computer Methods in Applied Mechanics and Engineering, 259, 40-49, 2013.

[IBA16] R. Ibanez, E. Abisset-Chavanne, J.V. Aguado, D. Gonzalez, E. Cueto, F. Chinesta F. A manifold-based methodological approach to data-driven computational elasticity and inelasticity. Archives of Computational Methods in Engineering, DOI 10.1007/s11831-0169197-9, 2016.

[LAD85] P. Ladeveze. On a family of algorithms for structural mechanics (in french). Comptes Rendus Académie des Sciences Paris, 300/2, 41-44, 1985.

[LAD89] P. Ladeveze, The large time increment method for the analyze of structures with nonlinear constitutive relation described by internal variables, Comptes Rendus Académie 
des Sciences Paris, 309, 1095-1099, 1989.

[LAD96] P. Ladeveze. Mécanique non linéaire des structures. Hermès, Paris, 1996.

[LAD99] P. Ladeveze. Nonlinear Computational structural mechanics. New approaches and non-incremental methods of calculation. Springer Verlag, 1999.

[LAM10] H. Lamari, A. Ammar, P. Cartraud, G. Legrain, F. Jacquemin, F. Chinesta. Routes for efficient computational homogenization of nonlinear materials using the Proper Generalized Decomposition. Archives of Computational Methods in Engineering, 17/4, 373-391, 2010.

[LEE07] J.A. Lee, M. Verleysen, Nonlinear dimensionality reduction. Springer, Berlin, 2007

[LOP15] E. Lopez, E. Abisset-Chavanne, S. Comas-Cardona, C. Binetruy, F. Chinesta. Flow modeling of linear and nonlinear fluids in two and three scale fibrous fabrics. International Journal of Material Forming. In press, DOI 10.1007/s12289-015-1224-0.

[LOP16] E. Lopez, D. Gonzalez, E. Abisset-Chavanne, E. Cueto, C. Binetruy, F. Chinesta. A manifold learning approach for integrated computational materials engineering. Archives of Computational Methods in Engineering, DOI 10.1007/s11831-016-9172-5, 2016.

[LOP16b] E. Lopez, A. Leygue, E. Abisset-Chavanne, S. Comas-Cardona, C. Aufrere, C. Binetruy, F. Chinesta. Flow modeling of linear and nonlinear fluids in two scale fibrous fabrics. Advanced simulations. International Journal of Material Forming, In press, DOI 10.1007/s12289-015-1280-5

[MAA08] L.J.P. van der Maaten and G.E. Hinton. Visualizing High-Dimensional Data Using t-SNE. Journal of Machine Learning Research, 9, 2579-2605, 2008

[NER10] D. Néron, P. Ladevèze, Proper generalized decomposition for multiscale and multiphysics problems, Archives of Computational Methods In Engineering, 17/4, 351-372, 2010.

[NER15] D. Neron, P.A. Boucard, N. Relun. Time-space PGD for the rapid solution of 3D nonlinear parametrized problems in the many-query context. International Journal for $\mathrm{Nu}$ merical Methods in Engineering,103/4, 275-292, 2015.

[NOU08] A. Nouy. Generalized spectral decomposition method for solving stochastic finite element equations : invariant subspace problem and dedicated algorithms. Computer Methods in Applied Mechanics and Engineering,197, 4718-4736, 2008.

[NOU09a] A. Nouy, O. Le Maitre. Generalized spectral decomposition method for stochastic non linear problems. Journal of Computational Physics, 228/1, :202-235, 2009.

[NOU09b] A. Nouy. Recent developments in spectral stochastic methods for the numerical solution of stochastic partial differential equations. Archives of Computational Methods in Engineering, 16/3, 251-285, 2009.

[NOU10] A. Nouy. Proper Generalized Decompositions and separated representations for the numerical solution of high dimensional stochastic problems. Archives of Computational Methods in Engineering - State of the Art Reviews, 17, 403-434, 2010.

[RIO13] H. Riou, P. Ladeveze, L. Kovalevsky. The Variational Theory of Complex Rays : An answer to the resolution of mid-frequency 3D engineering problems. J Sound Vib., 332, 1947-1960, 2013.

[ROW00] S.T. Roweis, L.K. Saul. Nonlinear dimensionality reduction by locally linear embedding. Science, 290/5500, 23232326, 2000

[VID12] P. Vidal, L. Gallimard, O. Polit. Composite beam finite element based on the Proper Generalized Decomposition. Computers \& Structures, 102, 76-86, 2012. 
[VID13] P. Vidal, L. Gallimard, O. Polit. Proper Generalized Decomposition and layer-wise approach for the modeling of composite plate structures. International Journal of Solids and Structures, 50/14-15, 2239-2250, 2013.

[ZHA04] Z. Zhang, H. Zha. Principal manifolds and nonlinear dimensionality reduction via tangent space alignement. SIAM J. Sci. Comput., 26/1, 313-338, 2004. 